\title{
Projection Continuation for Minimal Coordinate Set Dynamics of Constrained Systems
}

\author{
Ping Zhou ${ }^{1}$, Andrea Zanoni ${ }^{2}$, Pierangelo Masarati ${ }^{2}$ \\ ${ }^{1}$ School of Astronautics \\ ${ }^{2}$ Department of Aerospace Science and Technology \\ Politecnico di Milano \\ Harbin Institute of Technology \\ Harbin, China \\ ping.zhou@polimi.it \\ via La Masa 34, 20156, Milano, Italy \\ \{andrea.zanoni,pierangelo.masarati\}@ polimi.it
}

\begin{abstract}
The formulation of constrained system dynamics using coordinate projection onto a subspace locally tangent to the constraint manifold is revisited using the QR factorization of the constraint Jacobian matrix to extract a suitable subspace, and integrating the evolution of the QR factorization along with that of the constraint Jacobian matrix, as the solution evolves. A true continuation algorithm is thus proposed for the subspace of independent coordinates, which does not visibly affect the quality of the solution, but avoids the artificial algorithmic discontinuities in the generalized velocities that would result from arbitrary reparameterization of the coordinate set. This property is exemplified by solving simple multi-degree-of-freedom problems with and without the proposed continuation.
\end{abstract}

Keywords: Minimal Coordinate Set, Coordinate Projection, Automatic Coordinate Reduction, QR Factorization.

\section{INTRODUCTION}

In mechanical system dynamics, unconstrained dynamics problems are usually formulated as a set of second-order ordinary differential equations (ODE) that depend on a corresponding set of coordinates. A convenient approximation to describe the interaction between the parts of the system is often their idealization at a purely kinematic level, in form of algebraic relationships between the coordinates of the parts. The addition of these algebraic equations turns the problem into a system of differential-algebraic equations (DAE). The original coordinates are no longer independent; the actual number of independent coordinates reduces to that of the truly independent degrees of freedom. The enforcement of the constraints results in constraint reaction generalized forces, usually formulated as Lagrange multipliers. They represent the (unknown) internal forces whose value is whatever is required to guarantee the enforcement of the constraints. For a review of the possible approaches, see for example [1].

The constrained dynamics problem can be solved either directly, in form of a system of DAEs, where the original coordinates, augmented by the Lagrange multipliers, represent the unknowns in the so-called redundant coordinate set (RCS) formulation or, through manipulations that will be detailed in a later section, it can be transformed into the corresponding underlying ODE problem, reducing the set of coordinates to the truly independent (Lagrangian) ones, following the so-called minimal coordinate set (MCS) formulation. For a review of the possible approaches, see for example [2]. This paper focuses on this latter approach.

It is worth noticing that third approach is possible, i.e. to somehow embed the constraints in the unconstrained problem, formally preserving the original structure and unknowns. See for example the so-called Augmented Lagrangian approach [3] or the force projection method [4]. Also in this case, the problem formally reduces to ODE, with all the related implications, opportunities

\footnotetext{
${ }^{1}$ Ping Zhou is currently a visiting PhD student at Politecnico di Milano.
} 
and limitations in terms of approaches for its numerical integration: explicit methods can be used, subjected to conditional stability limitations.

It is recognized that the reduction of the original coordinates into the MCS may be a challenging task, and that their nature is local, i.e. there may not exist a generally valid choice, which works for all configurations of the system [5]. Being such choice local, when the coordinates need to be redefined with respect to a new configuration discontinuities in the generalized coordinates (specifically in their derivatives) are expected, although not related to any physical discontinuity in the kinematics or dynamics of the system. Indeed, the motion with respect to the original coordinates is not expected to show any discontinuity, the latter being mere artifacts of a redefinition of the local coordinates. This work presents a well-known and effective method for selecting a subspace of independent coordinates that is intrinsically tangent to the constraint manifold at a specific configuration, based on the QR factorization of the constraint Jacobian matrix, and discusses how to operate the redefinition of the coordinates' subspace in a continuous manner, to eliminate those unnecessary, formal discontinuities.

\section{PROBLEM DESCRIPTION}

\subsection{Constrained Dynamics Problem Formulation}

A generic constrained system dynamics problem is formulated by adding $m$ (holonomic, in the present case, and ideal) kinematic constraints, in form of the set of algebraic equations

$$
\mathbf{c}(\mathbf{x}, t)=\mathbf{0}
$$

with $\mathbf{c} \in \mathbb{R}^{m}$, to a set of $n$ ordinary differential equations ( $n \geq m$, but usually $n>m$ ) that express the dynamics of an unconstrained system of $n$ coordinates $\mathbf{x} \in \mathbb{R}^{n}$,

$$
\mathbf{M} \ddot{\mathbf{x}}=\mathbf{f}
$$

subjected to a set of generalized forces $\mathbf{f} \in \mathbb{R}^{n}$, energetically conjugated to a virtual perturbation of the coordinates, $\delta \mathbf{x}$. These equations are modified by the addition of the constraint reactions $\mathbf{f}_{c}=-\mathbf{c}_{/ \mathbf{x}}^{T} \lambda$, as

$$
\mathbf{M} \ddot{\mathbf{x}}+\mathbf{c}_{/ \mathbf{x}}^{T} \boldsymbol{\lambda}=\mathbf{f}
$$

where $\mathbf{c}_{/ \mathbf{x}}=\mathbf{A} \in \mathbb{R}^{m \times n}$ is the partial derivative of the constraint equations $\mathbf{c}$ with respect to the coordinates $\mathbf{x}$, namely the constraint Jacobian matrix, and $\lambda \in \mathbb{R}^{m}$ are the corresponding Lagrange multipliers.

\subsection{Minimal Coordinate Set Approach}

The Minimal Coordinate Set approach consists in defining a suitable subspace $\mathbf{T} \in \mathbb{R}^{n \times(n-m)}$ of the space spanned by the coordinates $\mathbf{x}$ which is tangent to the constraint manifold, namely $\mathbf{T}^{T} \mathbf{A}^{T} \equiv$ $\mathbf{0} \in \mathbb{R}^{(n-m) \times m}$, such that

$$
\begin{aligned}
& \dot{\mathbf{x}}=\mathbf{T} \dot{\mathbf{q}}+\boldsymbol{\beta}^{\prime} \\
& \ddot{\mathbf{x}}=\mathbf{T} \ddot{\mathbf{q}}+\boldsymbol{\beta}^{\prime \prime}
\end{aligned}
$$

where $\mathbf{q} \in \mathbb{R}^{n-m}$ are local, truly independent coordinates, with $\boldsymbol{\beta}^{\prime}$ and $\boldsymbol{\beta}^{\prime \prime}$ defined accordingly, the former being non-zero only in case of rheonomous constraints, such that

$$
\mathbf{c}_{/ \mathbf{x}} \dot{\mathbf{x}}+\mathbf{c}_{/ t}=\mathbf{0} \quad \rightarrow \quad \mathbf{c}_{/ \mathbf{x}}\left(\mathbf{T} \dot{\mathbf{q}}+\boldsymbol{\beta}^{\prime}\right)+\mathbf{c}_{/ t}=\mathbf{0} \quad \rightarrow \quad \mathbf{c}_{/ \mathbf{x}} \boldsymbol{\beta}^{\prime}=-\mathbf{c}_{/ t}
$$

being $\mathbf{c}_{/ \mathbf{x}} \mathbf{T}=\mathbf{A} \mathbf{T} \equiv \mathbf{0}$, and analogously

$$
\mathbf{c}_{/ \mathbf{x}} \ddot{\mathbf{x}}+(\dot{\mathbf{c}})_{/ \mathbf{x}} \dot{\mathbf{x}}+(\dot{\mathbf{c}})_{/ t}=\mathbf{0} \quad \rightarrow \quad \mathbf{c}_{/ \mathbf{x}} \boldsymbol{\beta}^{\prime \prime}=-(\dot{\mathbf{c}})_{/ \mathbf{x}} \dot{\mathbf{x}}-(\dot{\mathbf{c}})_{/ t}
$$


The constrained dynamics problem, projected in such subspace, yields

$$
\mathbf{T}^{T} \mathbf{M T} \ddot{\mathbf{q}}+\mathbf{T}^{T} \mathbf{A}^{T} \boldsymbol{\lambda}=\mathbf{T}^{T}\left(\mathbf{f}-\mathbf{M} \boldsymbol{\beta}^{\prime \prime}\right)
$$

The solution is sought by first integrating Eq. (7) to obtain the generalized velocities $\dot{\mathbf{q}}$; then, Eq. (4a) is integrated to obtain an estimate of $\mathbf{x}$, which needs to be subsequently refined by enforcing the constraint at the position level, Eq. (1).

\subsection{QR Factorization for Optimal Minimal Coordinate Set Selection}

Among the several approaches proposed in the literature [6], a suitable choice for $\mathbf{T}$ is obtained through the QR factorization [7] of the transpose of the constraint Jacobian matrix,

$$
\mathbf{A}^{T}=\mathbf{Q R}=\left[\begin{array}{ll}
\mathbf{Q}_{1} & \mathbf{Q}_{2}
\end{array}\right]\left[\begin{array}{c}
\mathbf{R}_{1} \\
\mathbf{0}
\end{array}\right]=\mathbf{Q}_{1} \mathbf{R}_{1}
$$

where matrix $\mathbf{Q} \in \mathbb{R}^{n \times n}$ is orthogonal and submatrix $\mathbf{R}_{1} \in \mathbb{R}^{m \times m}$ is upper triangular. Submatrix $\mathbf{Q}_{2} \in \mathbb{R}^{n \times(n-m)}$ represents an optimal choice for $\mathbf{T}$.

Velocities are then expressed as

$$
\dot{\mathbf{x}}=\mathbf{Q}_{2} \dot{\mathbf{q}}+\mathbf{Q}_{1} \mathbf{p}^{\prime}
$$

with $\mathbf{Q}_{1} \mathbf{p}^{\prime}=\boldsymbol{\beta}^{\prime}$, such that

$$
\mathbf{0}=\mathbf{A} \dot{\mathbf{x}}+\mathbf{c}_{/ t}=\mathbf{R}_{1}^{T} \mathbf{Q}_{1}^{T}\left(\mathbf{Q}_{2} \dot{\mathbf{q}}+\mathbf{Q}_{1} \mathbf{p}^{\prime}\right)+\mathbf{c}_{/ t}=\mathbf{R}_{1}^{T} \mathbf{p}^{\prime}+\mathbf{c}_{/ t} \quad \rightarrow \quad \mathbf{p}^{\prime}=-\mathbf{R}_{1}^{-T} \mathbf{c}_{/ t}
$$

whereas accelerations are expressed as

$$
\ddot{\mathbf{x}}=\mathbf{Q}_{2} \ddot{\mathbf{q}}+\mathbf{Q}_{1} \mathbf{p}^{\prime \prime}
$$

with $\mathbf{Q}_{1} \mathbf{p}^{\prime \prime}=\boldsymbol{\beta}^{\prime \prime}$, such that

$$
\begin{aligned}
\mathbf{0} & =\mathbf{A} \ddot{\mathbf{x}}+(\dot{\mathbf{c}})_{/ \mathbf{x}} \dot{\mathbf{x}}+(\dot{\mathbf{c}})_{/ t}=\mathbf{R}_{1}^{T} \mathbf{Q}_{1}^{T}\left(\mathbf{Q}_{2} \ddot{\mathbf{q}}+\mathbf{Q}_{1} \mathbf{p}^{\prime \prime}\right)+(\dot{\mathbf{c}})_{/ \mathbf{x}} \dot{\mathbf{x}}+(\dot{\mathbf{c}})_{/ t}=\mathbf{R}_{1}^{T} \mathbf{p}^{\prime \prime}+(\dot{\mathbf{c}})_{/ \mathbf{x}} \dot{\mathbf{x}}+(\dot{\mathbf{c}})_{/ t} \\
& \rightarrow \mathbf{p}^{\prime \prime}=-\mathbf{R}_{1}^{-T}\left[(\dot{\mathbf{c}})_{/ \mathbf{x}} \dot{\mathbf{x}}+(\dot{\mathbf{c}})_{/ t}\right]
\end{aligned}
$$

According to Eqs. (7) and (9), the problem becomes

$$
\begin{aligned}
\mathbf{Q}_{2}^{T} \mathbf{M} \mathbf{Q}_{2} \ddot{\mathbf{q}} & =\mathbf{Q}_{2}^{T}\left(\mathbf{f}-\mathbf{M} \boldsymbol{\beta}^{\prime \prime}\right) \\
\dot{\mathbf{x}} & =\mathbf{Q}_{2} \dot{\mathbf{q}}+\mathbf{Q}_{1} \mathbf{p}^{\prime}
\end{aligned}
$$

Its integration from time $t_{k}$ to $t_{k+1}$ yields

$$
\mathbf{x}_{k+1}^{(0)}=\mathbf{Q}_{2_{k}} \mathbf{q}_{k+1}+\mathbf{Q}_{1_{k}} \mathbf{p}^{(0)}
$$

where the subscript $(\cdot)^{(0)}$ indicates an estimate of the final value, pending verification that it complies with the constraint of Eq. (1). The final value of the unknown $\mathbf{p}$ results from the iterative solution of

$$
\mathbf{c}\left(\mathbf{x}_{k+1}, t_{k+1}\right)=\mathbf{0}
$$

namely

$$
\mathbf{c}\left(\mathbf{x}_{k+1}^{(i)}, t_{k+1}\right)+\left(\mathbf{c}_{/ \mathbf{x}}\right)_{k+1}^{(i)} \mathbf{Q}_{1_{k}} \Delta \mathbf{p}=\mathbf{0}
$$

i.e.

$$
\begin{aligned}
\Delta \mathbf{p} & =-\left(\left(\mathbf{c}_{/ \mathbf{x}}\right)_{k+1}^{(i)} \mathbf{Q}_{1_{k}}\right)^{-1} \mathbf{c}\left(\mathbf{x}_{k+1}^{(i)}, t_{k+1}\right) \\
\mathbf{p} & +=\Delta \mathbf{p}
\end{aligned}
$$

where $\left(\mathbf{c}_{/ \mathbf{x}}\right)_{k+1}^{(i)}$ is the constraint Jacobian matrix at time $t_{k+1}$ during the $i$ th constraint enforcement iteration, evaluated as a function of $\mathbf{x}_{k+1}^{(i)}$. 


\subsection{Tangent Subspace Selection and Continuation}

Submatrices $\mathbf{Q}_{1}$ and $\mathbf{R}_{1}$ are uniquely determined ${ }^{1}$, once $\mathbf{A}$ is known. Submatrix $\mathbf{Q}_{2}$, instead, is only subjected to matrix $\mathbf{Q}$ 's general constraint of being orthogonal, namely $\mathbf{Q}_{2}^{T} \mathbf{Q}_{2} \equiv \mathbf{I} \in \mathbb{R}^{(n-m) \times(n-m)}$ and $\mathbf{Q}_{2}^{T} \mathbf{Q}_{1} \equiv \mathbf{0} \in \mathbb{R}^{(n-m) \times m}$, but otherwise undefined. Specifically, it is defined in excess of postmultiplication by an arbitrary orthogonal matrix, $\mathbf{P} \in \mathbb{R}^{(n-m) \times(n-m)}: \tilde{\mathbf{Q}}_{2}=\mathbf{Q}_{2} \mathbf{P}$ also complies with the orthogonality requirement, since $\tilde{\mathbf{Q}}_{2}^{T} \tilde{\mathbf{Q}}_{2}=\mathbf{P}^{T} \mathbf{Q}_{2}^{T} \mathbf{Q}_{2} \mathbf{P}=\mathbf{P}^{T} \mathbf{P} \equiv \mathbf{I}$ and $\tilde{\mathbf{Q}}_{2}^{T} \mathbf{Q}_{1}=\mathbf{P}^{T} \mathbf{Q}_{2}^{T} \mathbf{Q}_{1}=$ $\mathbf{P}^{T} \mathbf{0}=\mathbf{0}$.

In fact, the QR factorization produces a "local" representation of the constraint Jacobian matrix; as such, the generalized coordinates associated with the subspace $\mathbf{T}=\mathbf{Q}_{2}$, which do not have any specific physical meaning, represent a local reparameterization of the subspace of the coordinates that is tangent to the constraint manifold. When the $\mathrm{QR}$ factorization is computed at different time steps $t_{k}$, if $n-m>1$ the columns of the resulting $\mathbf{Q}_{2_{k}}$ are completely unrelated, their resulting value being solely dictated by the internal intricacies of the $\mathrm{QR}$ factorization algorithm.

The aim of the present work is to propose a simple and intuitive algorithm that tracks the evolution of the subspace spanned by $\mathbf{Q}_{2}$ using a form of differential "continuation," to preserve some sort of spatial continuity of the generalized coordinates, $\mathbf{q}$, by minimizing the amount of deviation of the subspace that is intrinsically required to maintain $\mathbf{Q}_{2}$ tangent to the constraint manifold across time steps, without altering the quality of the solution.

Consider the time derivative of the transpose of the constraint Jacobian matrix in its QR factorized form,

$$
\dot{\mathbf{A}}^{T}=\dot{\mathbf{Q}} \mathbf{R}+\mathbf{Q} \dot{\mathbf{R}}
$$

The derivative of matrix $\mathbf{Q}$ may be expressed as $\dot{\mathbf{Q}}=\mathbf{Q} \boldsymbol{\Omega}$, where the skew-symmetric nature of matrix $\boldsymbol{\Omega} \in \mathbb{R}^{n \times n}$ descends from the orthogonality of matrix $\mathbf{Q}$ :

$$
\frac{\mathrm{d}}{\mathrm{d} t}\left(\mathbf{Q}^{T} \mathbf{Q}\right)=\dot{\mathbf{Q}}^{T} \mathbf{Q}+\mathbf{Q}^{T} \dot{\mathbf{Q}}=\left(\mathbf{Q}^{T} \dot{\mathbf{Q}}\right)^{T}+\mathbf{Q}^{T} \dot{\mathbf{Q}}=\mathbf{0} \quad \rightarrow \quad \mathbf{Q}^{T} \dot{\mathbf{Q}}=\mathbf{\Omega}
$$

When the problem is integrated numerically, the solution from time step $t_{k}$ to time step $t_{k+1}$ is computed. The QR factorization at time $t_{k}$ yields submatrices $\mathbf{Q}_{1_{k}}$ and $\mathbf{R}_{1_{k}}$. The generalized velocities at time $t_{k}$ are computed with reference to the subspace spanned by $\mathbf{Q}_{2_{k}}$. After computing the solution at the new time step, the Jacobian matrix at time $t_{k+1}, \mathbf{A}_{k+1}$, is known. As such, through the economy QR factorization of its transpose, submatrices $\mathbf{Q}_{1_{k+1}}$ and $\mathbf{R}_{1_{k+1}}$ are determined. Instead of computing also submatrix $\mathbf{Q}_{2_{k+1}}$ through the full $\mathrm{QR}$ factorization, the proposed continuation algorithm is used as illustrated in the following. Consider

$$
\mathbf{Q}_{1}^{T} \dot{\mathbf{A}}^{T} \mathbf{R}_{1}^{-1}=\mathbf{Q}_{1}^{T} \dot{\mathbf{Q}}_{1}+\dot{\mathbf{R}}_{1} \mathbf{R}_{1}^{-1}
$$

Matrix $\dot{\mathbf{R}}_{1} \mathbf{R}_{1}^{-1}$ is the product of two upper triangular matrices, thus it is itself an upper triangular matrix. Matrix $\mathbf{Q}_{1}^{T} \dot{\mathbf{Q}}_{1}=\boldsymbol{\Omega}_{1} \in \mathbb{R}^{m \times m}$ is skew-symmetric by construction; it can be seen as $\boldsymbol{\Omega}_{1}=$ $\boldsymbol{\Omega}_{1_{L}}-\boldsymbol{\Omega}_{1_{L}}^{T}$, where $\boldsymbol{\Omega}_{1_{L}}=\operatorname{stril}\left(\boldsymbol{\Omega}_{1}\right)$ is the strictly lower triangular part of matrix $\boldsymbol{\Omega}_{1}$, which can be obtained as

$$
\boldsymbol{\Omega}_{1_{L}}=\operatorname{stril}\left(\mathbf{Q}_{1}^{T} \dot{\mathbf{A}}^{T} \mathbf{R}_{1}^{-1}\right)
$$

since stril $\left(\dot{\mathbf{R}}_{1} \mathbf{R}_{1}^{-1}\right) \equiv \mathbf{0}$ by construction, being $\dot{\mathbf{R}}_{1} \mathbf{R}_{1}^{-1}$ upper triangular. From Eq. (20), one can show that the derivative of matrix $\mathbf{Q}$,

$$
\begin{aligned}
\dot{\mathbf{Q}}=\left[\begin{array}{ll}
\dot{\mathbf{Q}}_{1} & \dot{\mathbf{Q}}_{2}
\end{array}\right] & =\left[\begin{array}{ll}
\mathbf{Q}_{1} & \mathbf{Q}_{2}
\end{array}\right]\left[\begin{array}{cc}
\boldsymbol{\Omega}_{1} & -\mathbf{R}_{1}^{-T} \dot{\mathbf{A}} \mathbf{Q}_{2} \\
\mathbf{Q}_{2}^{T} \dot{\mathbf{A}}^{T} \mathbf{R}_{1}^{-1} & \mathbf{\Omega}_{2}
\end{array}\right] \\
& =\left[\begin{array}{ll}
\mathbf{Q}_{1} & \mathbf{Q}_{2}
\end{array}\right]\left[\begin{array}{cc}
\mathbf{\Omega}_{1} & -\boldsymbol{\Omega}_{21}^{T} \\
\mathbf{\Omega}_{21} & \mathbf{0}
\end{array}\right]=\mathbf{Q} \boldsymbol{\Omega}
\end{aligned}
$$

\footnotetext{
${ }^{1}$ To this end, we choose the diagonal elements of $\mathbf{R}_{1}$ to be non-negative, a zero value indicating indetermination.
} 
is entirely known, where the bottom right block of the rightmost matrix, $\boldsymbol{\Omega}$, should contain an unknown skew-symmetric contribution $\boldsymbol{\Omega}_{2} \in \mathbb{R}^{(n-m) \times(n-m)}$, that is arbitrarily set to zero to modify as little as possible the subspace $\mathbf{Q}_{2}$; specifically,

$$
\dot{\mathbf{Q}}_{2}=-\mathbf{Q}_{1} \boldsymbol{\Omega}_{21}^{T}=-\mathbf{A}^{+} \dot{\mathbf{A}} \mathbf{Q}_{2}
$$

In fact, $\boldsymbol{\Omega}_{2}$ may be interpreted as the angular velocity of subspace $\mathbf{Q}_{2}$, the rate of reorientation with respect to itself, whereas $\boldsymbol{\Omega}_{12}$ expresses the rate of reorientation with respect to $\mathbf{Q}_{1}$, in order to remain orthogonal to it.

Thus, the subspace $\mathbf{Q}_{2}$ can be integrated, taking appropriate measures (e.g. using Munthe-Kaas' method [8]) to guarantee that the resulting matrix $\mathbf{Q}$ preserves orthogonality, and submatrix $\mathbf{Q}_{1}$ matches that resulting from the decomposition of the transpose of the constraint Jacobian matrix. For example, for $\boldsymbol{\Omega}$ constant across a time step of duration $t_{k+1}-t_{k}=h$,

$$
\mathbf{Q}_{k+1}=\mathbf{Q}_{k} \mathrm{e}^{\mathbf{\Omega} h}
$$

or

$$
\mathbf{Q}_{2_{k+1}}=\mathrm{e}^{-\mathbf{A}^{+} \dot{\mathbf{A}} h} \mathbf{Q}_{2_{k}}
$$

the latter being only a first-order approximation of the former, since the intrinsic skew-symmetric structure of the exponent matrix $\boldsymbol{\Omega}$ is lost.

Submatrix $\mathbf{Q}_{2_{k+1}}$ resulting from the proposed integration may need to be corrected to guarantee orthogonality with respect to submatrix $\mathbf{Q}_{1_{k+1}}$ obtained from the economy QR factorization of $\mathbf{A}_{k+1}^{T}$.

\section{RESULTS}

Two simple examples, a spatial (3D) pendulum and a spin top, are analyzed to illustrate how the proposed method produces a more regular and intuitive choice of the projection subspace during the integration of the solution.

Spatial Pendulum Consider a simple point mass spherical pendulum of mass $M$ and length $\ell$, subjected to a uniform gravity field $g=9.81 \mathrm{~m} / \mathrm{s}^{2}$, directed along the negative $z$ axis. Its equations of motion are

$$
\begin{gathered}
{\left[\begin{array}{ccc}
M & 0 & 0 \\
0 & M & 0 \\
0 & 0 & M
\end{array}\right]\left\{\begin{array}{l}
\ddot{x} \\
\ddot{y} \\
\ddot{z}
\end{array}\right\}+\left[\begin{array}{c}
2 x \\
2 y \\
2 z
\end{array}\right] \lambda=\left\{\begin{array}{c}
0 \\
0 \\
-M g
\end{array}\right\}} \\
x^{2}+y^{2}+z^{2}-\ell^{2}=0
\end{gathered}
$$

The unconstrained problem has 3 degrees of freedom, $x, y$ and $z$, and 1 constraint, Eq. (27a); thus, the constrained problem has 2 degrees of freedom. Consequently, $\mathbf{Q}_{1} \in \mathbb{R}^{3 \times 1}$ and $\mathbf{Q}_{2} \in \mathbb{R}^{3 \times 2}$. The constraint Jacobian matrix and its time derivative are

$$
\begin{aligned}
\mathbf{A} & =\left[\begin{array}{lll}
2 x & 2 y & 2 z
\end{array}\right] \\
\dot{\mathbf{A}} & =\left[\begin{array}{lll}
2 \dot{x} & 2 \dot{y} & 2 \dot{z}
\end{array}\right]
\end{aligned}
$$

The QR factorization of $\mathbf{A}^{T}$ in a given initial configuration $(x, y, z)=\left(x_{0}, y_{0}, z_{0}\right)$ yields

$$
\mathbf{A}^{T}=\left[\begin{array}{c}
2 x_{0} \\
2 y_{0} \\
2 z_{0}
\end{array}\right]=\left[\begin{array}{c|cc}
x_{0} / \ell & q_{12} & q_{13} \\
y_{0} / \ell & q_{22} & q_{23} \\
z_{0} / \ell & q_{32} & q_{33}
\end{array}\right]\left[\begin{array}{c}
2 \ell \\
\hline 0 \\
0
\end{array}\right]=\mathbf{Q R}
$$


where the 6 coefficients $q_{i j}, i=1,2,3, j=2,3$ are related by 5 orthogonality conditions, leaving only one undetermined parameter.

Without loss of generality, let us assume that $\left(x_{0}, y_{0}, z_{0}\right)=(\ell, 0,0)$, which complies with the constraint equation; this yields

$$
\mathbf{A}^{T}=\left[\begin{array}{c}
2 \ell \\
0 \\
0
\end{array}\right]=\left[\begin{array}{c|cc}
1 & 0 & 0 \\
0 & \cos \alpha_{0} & -\sin \alpha_{0} \\
0 & \sin \alpha_{0} & \cos \alpha_{0}
\end{array}\right]\left[\begin{array}{c}
2 \ell \\
0 \\
0
\end{array}\right]=\mathbf{Q R}
$$

where $\alpha_{0}$ is an arbitrary parameter. Clearly, when $\alpha_{0}=0$, the two vectors that span the subspace of $\mathbf{Q}_{2}$ are the coordinate axes $y$ and $z$.

The projected equations of motion in the initial configuration are:

$$
\left[\begin{array}{cc}
M & 0 \\
0 & M
\end{array}\right]\left\{\begin{array}{l}
\ddot{q}_{1} \\
\ddot{q}_{2}
\end{array}\right\}=\left\{\begin{array}{l}
-\sin \alpha_{0} \\
-\cos \alpha_{0}
\end{array}\right\} M g
$$

Without loss of generality, it is assumed that $\left(\dot{x}_{0}, \dot{y}_{0}, \dot{z}_{0}\right)=\left(0, v_{0}, 0\right)$, which complies with the derivative of the constraint equation in the initial configuration,

$$
0=\mathbf{A} \dot{\mathbf{x}}=\left[\begin{array}{ccc}
2 \ell & 0 & 0
\end{array}\right]\left\{\begin{array}{c}
0 \\
v_{0} \\
0
\end{array}\right\}
$$

in this case, one obtains

$$
\begin{aligned}
& \boldsymbol{\Omega}_{1_{L}}=0 \text { [as one would expect for a diagonal element of a skew-symmetric matrix] } \\
& \boldsymbol{\Omega}_{21}=\left[\begin{array}{ccc}
0 & \cos \alpha_{0} & \sin \alpha_{0} \\
0 & -\sin \alpha_{0} & \cos \alpha_{0}
\end{array}\right]\left[\begin{array}{c}
0 \\
2 v_{0} \\
0
\end{array}\right] \frac{1}{2 \ell}=\left[\begin{array}{c}
\cos \alpha_{0} \\
-\sin \alpha_{0}
\end{array}\right] \frac{v_{0}}{\ell}
\end{aligned}
$$

thus

$$
\boldsymbol{\Omega}=\frac{v_{0}}{\ell}\left[\begin{array}{ccc}
0 & -\cos \alpha_{0} & \sin \alpha_{0} \\
\cos \alpha_{0} & 0 & 0 \\
-\sin \alpha_{0} & 0 & 0
\end{array}\right]
$$

and

$$
\Delta \mathbf{Q}=\left[\begin{array}{ccc}
\cos \Delta \theta & -\sin \Delta \theta \cos \alpha_{0} & \sin \Delta \theta \sin \alpha_{0} \\
\sin \Delta \theta \cos \alpha_{0} & \cos \Delta \theta \cos \alpha_{0}^{2}-\cos \alpha_{0}^{2}+1 & -\sin \left(2 \alpha_{0}\right)(\cos \Delta \theta-1) / 2 \\
-\sin \Delta \theta \sin \alpha_{0} & -\sin \left(2 \alpha_{0}\right)(\cos \Delta \theta-1) / 2 & \cos \Delta \theta+\cos \alpha_{0}^{2}-\cos \Delta \theta \cos \alpha_{0}^{2}
\end{array}\right]
$$

with $\Delta \theta=v_{0} h / \ell$ which, for $\alpha_{0}=0$, reduces to

$$
\Delta \mathbf{Q}=\left[\begin{array}{ccc}
\cos \Delta \theta & -\sin \Delta \theta & 0 \\
\sin \Delta \theta & \cos \Delta \theta & 0 \\
0 & 0 & 1
\end{array}\right]
$$

namely, a finite rotation about the $z$ axis by an angle $\Delta \theta$.

In the present example, the mass of the pendulum is $M=1.0 \mathrm{~kg}$, and its length is $\ell=0.16 \mathrm{~m}$. The initial position of the mass center is $\mathbf{r}_{0}=[0.08,0,0]^{T} \mathrm{~m}$, and the initial condition is set as $v_{0}=0.7895 \mathrm{~m} / \mathrm{s}$. 


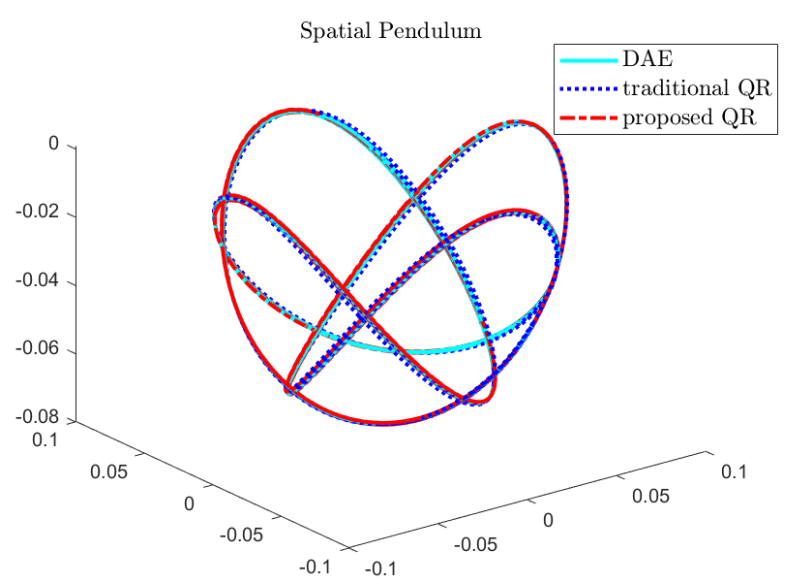

Figure 1. Trajectory of the spatial pendulum's center of mass: traditional and proposed QR approach results are compared to those obtained from DAE integration using MBDyn.
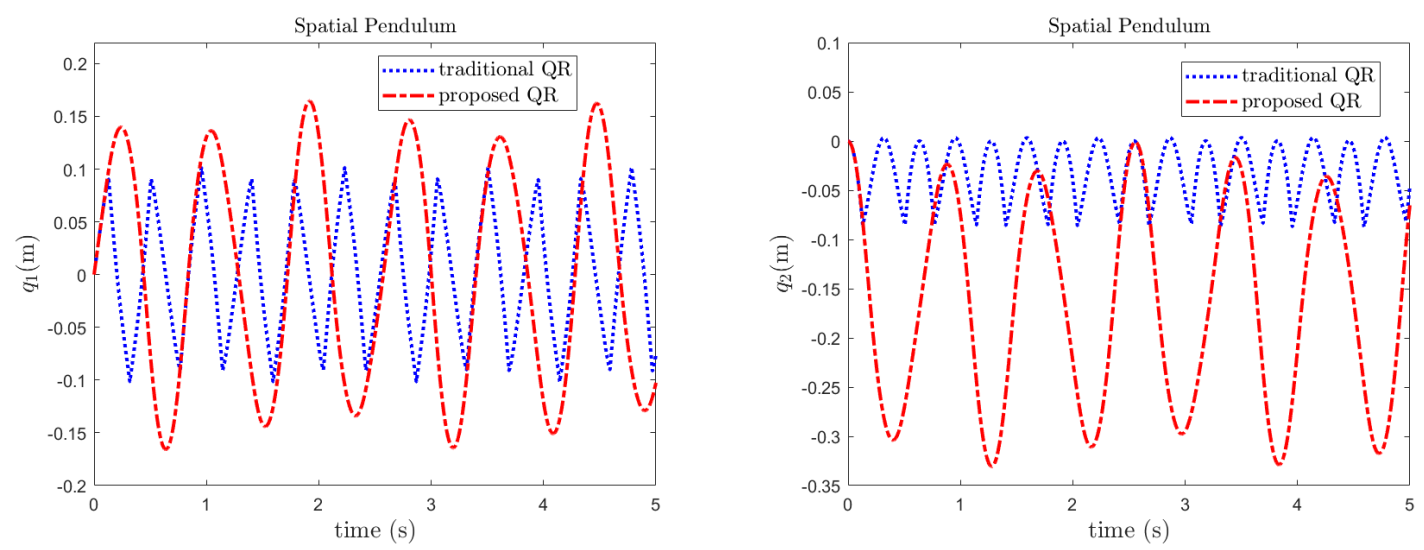

Figure 2. Minimal coordinates $q_{i},(i=1,2)$ : comparison of traditional and proposed QR factorization method.

The trajectory of its center of mass resulting from the simulation using ode45 in Matlab with $h=0.001 \mathrm{~s}$ is compared to that resulting from the integration of the original DAE governing equations using the free general-purpose multibody solver $\mathrm{MBDyn}^{2}$ [9], using a second-order accurate implicit linear multistep integration method with algorithmic dissipation (asymptotic spectral radius $\rho_{\infty}=0.6$ ) [10], and a projection method based on the QR factorization of the transpose of the Jacobian matrix performed at each time step, without any knowledge of its evolution, using ode45 with a time step $h=0.000005$ s to act as a reference solution, as shown in Fig. 1 . The resulting minimal set generalized coordinates $q_{1}$ and $q_{2}$ and the projected generalized velocities $\dot{q}_{1}$ and $\dot{q}_{2}\left(\dot{q}_{i}=\mathbf{Q}_{2}(:, i)^{T} \dot{\mathbf{x}}\right)$ are compared to the results obtained from what is here termed "traditional QR method", as shown in Fig. 2 and Fig. 3, respectively. One may observe that the coordinates $q_{i}$ resulting from the proposed method are much more regular than those resulting from the traditional QR factorization. Specifically, those resulting from the proposed method appear to be continuous and differentiable, whereas those resulting from the traditional QR factorization show discontinuities in their first derivatives, $\dot{q}_{i}$.

This is well explained by the continuity and regularity of the evolution of each column of matrix

\footnotetext{
${ }^{2}$ https://www . mbdyn .org/.
} 

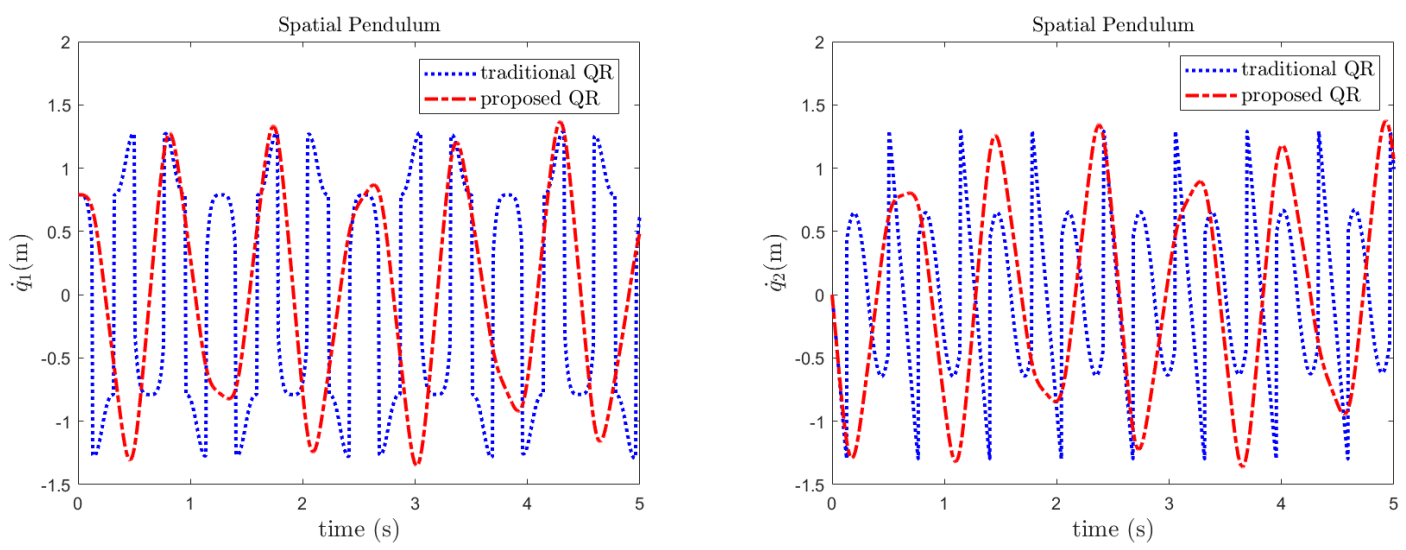

Figure 3. Projected velocities $\dot{q}_{i}(i=1,2)$ : comparison of traditional and proposed QR factorization method.

$\mathbf{Q}_{2}$ for the proposed method, compared to the discontinuity of those resulting from the traditional QR factorization, as depicted in Fig. 4.

Furthermore, from Fig. 4 one can observe that for $t=0$, the first column of matrix $\mathbf{Q}_{2}$ corresponds to $[0,1,0]^{T}$, i.e. the unit vector along the $y$ axis, whereas the second column of matrix $\mathbf{Q}_{2}$ corresponds to $[0,0,1]^{T}$, i.e. the unit vector along the $z$ axis, i.e. the $\mathrm{QR}$ algorithm chose $\alpha_{0}=0$ in Eq. (31) when initializing the subspace $\mathbf{Q}_{2}$. Indeed, considering the initial velocity of the spin top, one can observe that $\dot{q}_{1}(0) \equiv v_{0}$ and $\dot{q}_{2}(0) \equiv 0$, which is consistent with the given initial conditions. At time $t=0, \dot{q}_{1}$ corresponds to $v$, and $\dot{q}_{2}$ to $w$.

Spin Top Consider a symmetric spin top, whose tip is constrained to be at unit distance from the origin of the global coordinate system, i.e. lying on a sphere of unit radius centered in the origin. The tip of the spin top is $1 \mathrm{~m}$ far away from its center of mass. The problem is sketched in Fig. 5. The inertia properties of the spin top are $m=30 \mathrm{~kg}$ and $\mathbf{J}=\operatorname{diag}(90,90,30) \mathrm{kg} \cdot \mathrm{m}^{2}$. The initial position of the spin top is $\mathbf{r}_{0}=[0,-1,0]^{T} \mathrm{~m}$. The local coordinate system $x^{\prime}-y^{\prime}-z^{\prime}$ is initially coincident with the global coordinate system $x-y-z$. A uniform gravity field of magnitude $9.81 \mathrm{~m} / \mathrm{s}^{2}$ is assumed in the negative $z$ direction. In this model, the Euler parameters e are used to describe the rotation of the spin top; their initial value is $\mathbf{e}_{0}=[1,0,0,0]^{T}$. The initial velocity is $\dot{\mathbf{r}}_{0}=\mathbf{0} \mathrm{m} / \mathrm{s}$ and the initial angular velocity is $\omega_{0}=[0.1,0.1,0.3]^{T} \mathrm{rad} / \mathrm{s}$. The initial derivative of the Euler parameters $\dot{\mathbf{e}}_{0}$ is computed from $1 / 2 \cdot \mathbf{E}\left(\mathbf{e}_{0}\right) \omega_{0}$. The constrained equations of motion of the spin top are

$$
\begin{aligned}
m \ddot{\mathbf{r}}+c_{\mathbf{r}}^{T} \lambda & =\mathbf{f} \\
4 \mathbf{E}^{T} \mathbf{J E} \ddot{\mathbf{e}}+8 \dot{\mathbf{E}}^{T} \mathbf{J E} \omega+c_{\mathbf{e}}^{T} \lambda+2 \mathbf{e}^{T} \mu & =2 \mathbf{E}^{T} \mathbf{t} \\
c(t, \mathbf{r}, \mathbf{e}) & =0 \\
\mathbf{e}^{T} \mathbf{e}-1 & =0
\end{aligned}
$$

where $\mathbf{f}$ and $\mathbf{t}$ are the applied force and torque at the mass center, respectively. Since only gravity is applied, $\mathbf{f}=(0,0,-9.81 \mathrm{~m})$ and $\mathbf{t}=\mathbf{0}$, with

$$
\mathbf{E}=\left(\begin{array}{cccc}
-e_{1} & e_{0} & e_{3} & -e_{2} \\
-e_{2} & -e_{3} & e_{0} & e_{1} \\
-e_{3} & e_{2} & -e_{1} & e_{0}
\end{array}\right)
$$

$c$ denotes the constraint equation, $\lambda$ and $\mu$ are the Lagrange multipliers corresponding to the constraint equation $c$, Eq. (39c), and the Euler parameter normalization, Eq. (39d), respectively. 

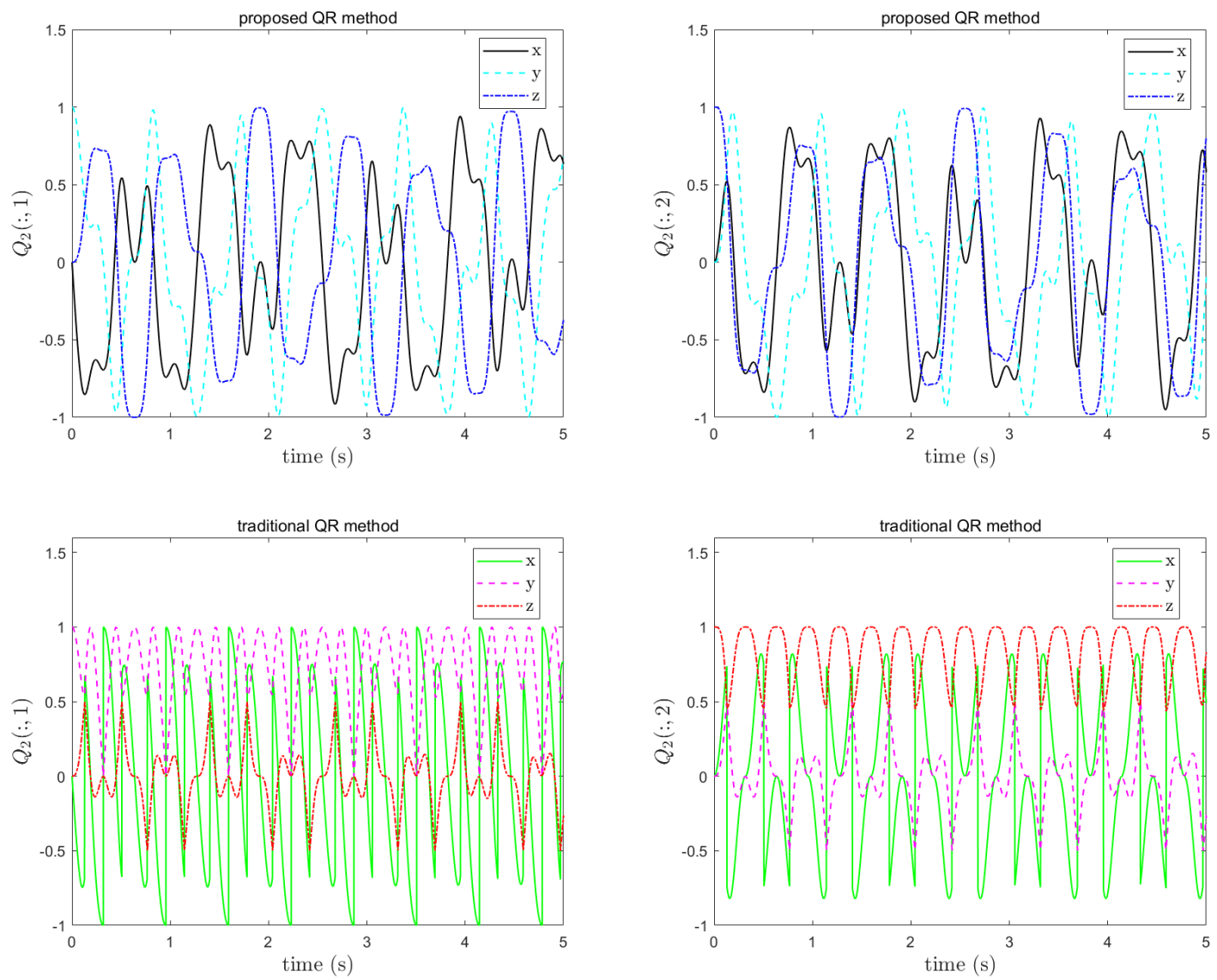

Figure 4. $\mathbf{Q}_{2}(:, i)(i=1,2)$ from the proposed (top) and the traditional QR method (bottom).

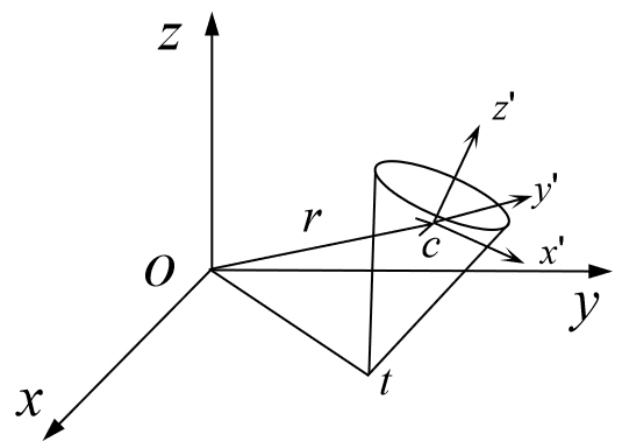

Figure 5. Spin top. 


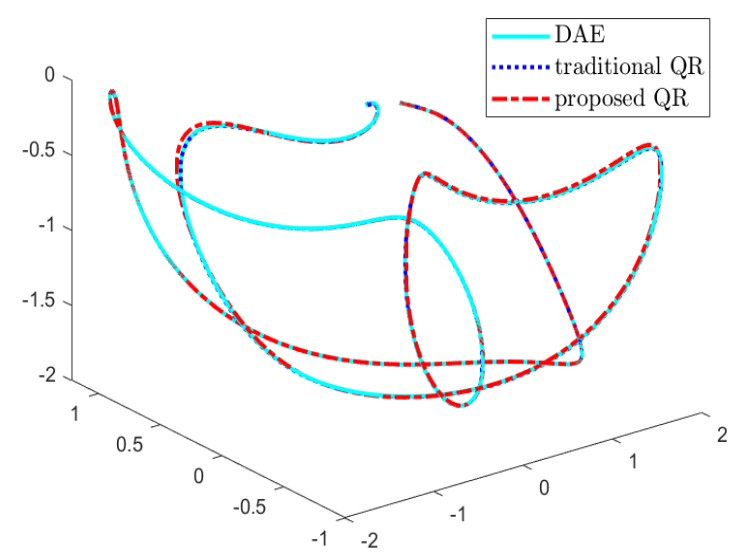

Figure 6. Trajectory of the spin top's center of mass: traditional and proposed QR approach results are compared to those obtained from DAE integration using MBDyn.

The absolute position of the tip is $\mathbf{r}_{t}=\mathbf{r}+\mathbf{A} \mathbf{u}_{0}$, where $\mathbf{u}_{0}=[0,0,-1]^{T} \mathrm{~m}$ and $\mathbf{A}=\overline{\mathbf{E}} \mathbf{E}^{T}$ is the orientation matrix, with

$$
\overline{\mathbf{E}}=\left(\begin{array}{cccc}
-e_{1} & e_{0} & -e_{3} & e_{2} \\
-e_{2} & e_{3} & e_{0} & -e_{1} \\
-e_{3} & -e_{2} & e_{1} & e_{0}
\end{array}\right)
$$

Therefore, the constraint equation can be expressed as

$$
c=\mathbf{r}_{t}^{T} \mathbf{r}_{t}-1=\mathbf{r}^{T} \mathbf{r}+2 \mathbf{r}^{T} \mathbf{A} \mathbf{u}_{0}
$$

since $\mathbf{u}_{0}^{T} \mathbf{u}_{0} \equiv 1$. The problem is described by 7 coordinates, $\mathbf{r} \in \mathbb{R}^{3}$ and $\mathbf{e} \in \mathbb{R}^{4}$, and 2 constraint equations, Eqs. (40) and (39d), thus possesses 5 degrees of freedom, $q_{i}(i=1, \ldots, 5)$.

The trajectory of the centroid resulting from the simulation using the previously mentioned method with $h=0.0001 \mathrm{~s}$ is compared in Fig. 6 to those obtained by integrating the original DAE system using MBDyn and by using the traditional QR method, with $h=0.0001 \mathrm{~s}$. The projection motion of $q_{i}(i=1, \ldots, 5)$ is compared to the results of the traditional QR method without any projection in Fig. 7, whereas that of $\dot{q}_{i}(i=1, \ldots, 5)$ is compared to the results of the traditional QR method without any projection in Fig. 8. Again, one can notice the much greater regularity of the coordinates and their derivatives as they result from the proposed method.

\section{CONCLUSIONS}

This paper presented a continuation algorithm for the redefinition of the subspace of minimal coordinates that is tangent to the constraint manifold. It is based on the full QR factorization of the constraint Jacobian matrix to initialize the subspace through the portion of the space defined by the orthogonal matrix $\mathrm{Q}$ that is orthogonal to the constraint Jacobian matrix. The economy QR factorization is then used to exactly factor the subspace in which the constraint Jacobian lies, while evolution of the tangent subspace is tracked by integrating the time derivative of matrix Q, eventually re-orthogonalizing the result to eliminate possible drift from the integrated tangent subspace. Numerical examples show that the result of the analysis is unchanged, but the generalized velocities do not show the discontinuities that characterize them when the tangent subspace is recomputed without considering its previous value. 

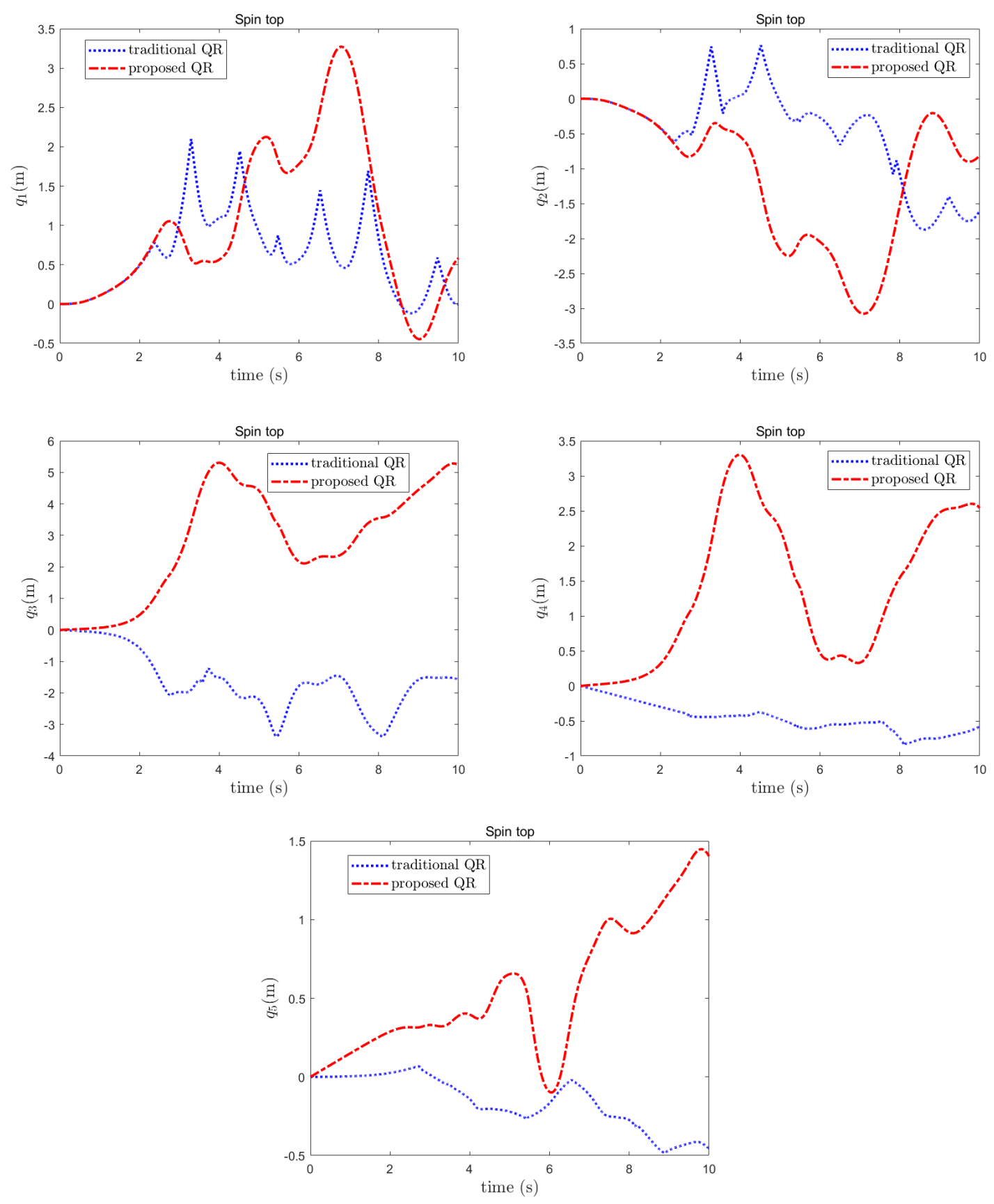

Figure 7. Minimal coordinates $q_{i}(i=1, \ldots, 5)$ : comparison of traditional and proposed QR factorization method. 

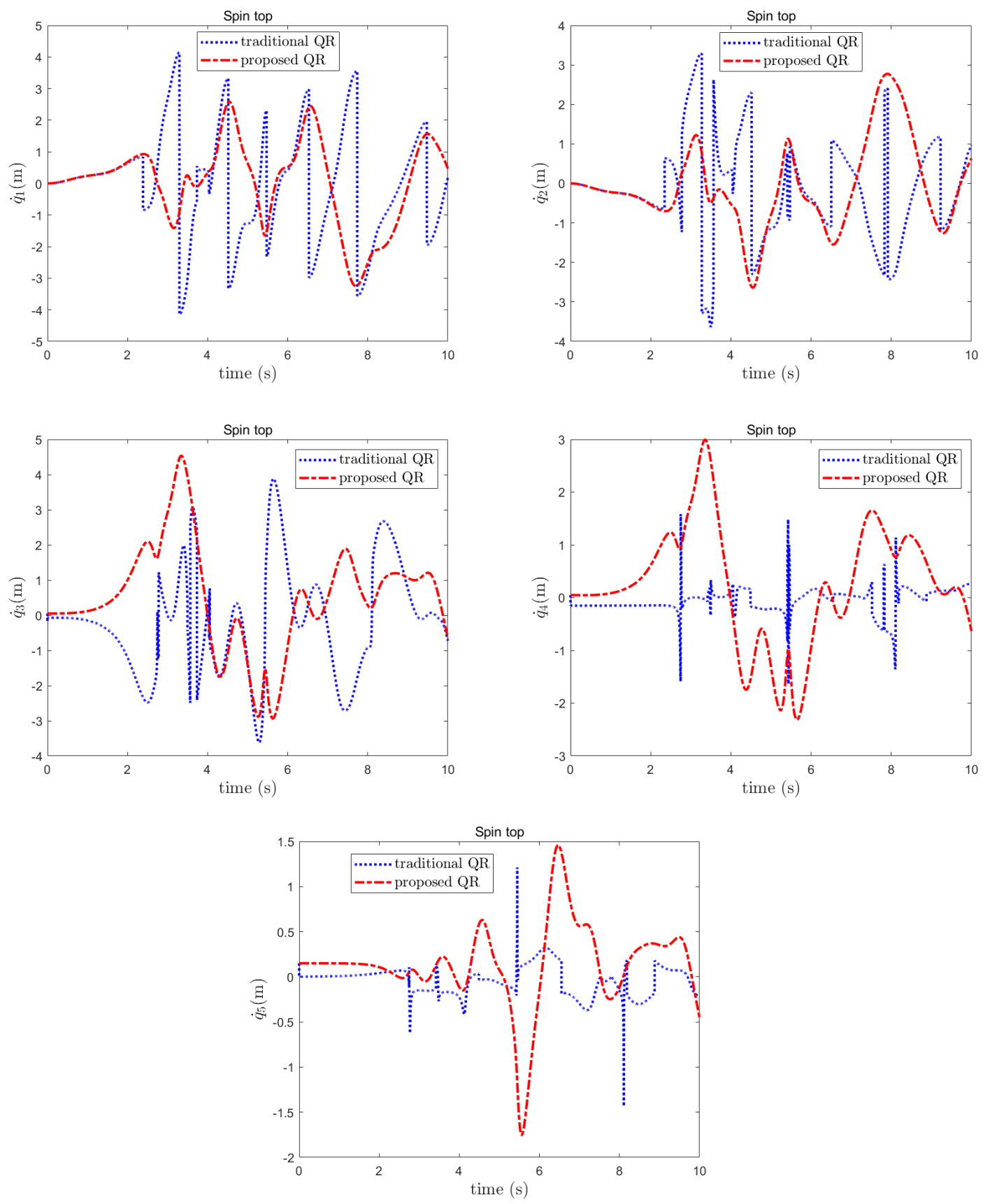

Figure 8. Projected velocities $\dot{q}_{i}(i=1, \ldots, 5)$ : comparison of traditional and proposed QR factorization method. 


\section{ACKNOWLEDGMENTS}

The first author acknowledges support from the China Scholarship Council.

\section{REFERENCES}

[1] Bauchau, O.A., Laulusa, A.: Review of contemporary approaches for constraint enforcement in multibody systems. J. of Computational and Nonlinear Dynamics 3(1) (January 2008) doi:10.1115/1.2803258.

[2] Laulusa, A., Bauchau, O.A.: Review of classical approaches for constraint enforcement in multibody systems. J. of Computational and Nonlinear Dynamics 3(1) (January 2008) doi:10.1115/1.2803257.

[3] Bayo, E., de Jalón, J.G., Serna, M.A.: A modified Lagrangian formulation for the dynamic analysis of constrained mechanical systems. Comput. Meth. Appl. Mech. Engng. 71(2) (1988) 183-195 doi:10.1016/0045-7825(88)90085-0.

[4] Masarati, P.: Adding kinematic constraints to purely differential dynamics. Computational Mechanics 47(2) (2011) 187-203 doi:10.1007/s00466-010-0539-4.

[5] Haug, E.J.: Multibody Dynamics on Differentiable Manifolds. J. of Computational and Nonlinear Dynamics 16(4) (02 2021) 041003 doi:10.1115/1.4049995.

[6] Mariti, L., Belfiore, N.P., Pennestrì, E., Valentini, P.P.: Comparison of solution strategies for multibody dynamics equations. Intl. J. Num. Meth. Engng. (2011) doi:10.1002/nme.3190.

[7] Golub, G.H., Van Loan, C.F.: Matrix Computations. 3rd edn. The Johns Hopkins University Press, Baltimore and London (1996)

[8] Munthe-Kaas, H.: High order Runge-Kutta methods on manifolds. Applied Numerical Mathematics 29(1) (1999) 115-127 doi:10.1016/S0168-9274(98)00030-0.

[9] Masarati, P., Morandini, M., Mantegazza, P.: An efficient formulation for general-purpose multibody/multiphysics analysis. J. of Computational and Nonlinear Dynamics 9(4) (2014) 041001 doi:10.1115/1.4025628.

[10] Zhang, H., Zhang, R., Masarati, P.: Improved second-order unconditionally stable schemes of linear multi-step and equivalent single-step integration methods. Computational Mechanics (2021) doi:10.1007/s00466-020-01933-y. 